Pacific Journal of Mathematics

CONSTRUCTION OF A CLASS OF MODULAR FUNCTION 


\title{
CONSTRUCTION OF A CLASS OF MODULAR FUNCTIONS AND FORMS II
}

\author{
MARVIN ISADORE KNOPP
}

To the memory of Mr. Adolph Zaidovsky

1. Introduction. In the previous paper [4], the author introduced functions, defined in $\mathscr{J}(\tau)>0$, which were shown to possess transformation properties under $G(j)$, the principal congruence subgroup, of level $j$, of the modular group $(j \geqq 2)$. The case $j=2$ is slightly different from the others and for the sake of simplicity we defer its treatment until $\S 5$.

With a fixed $j \geqq 3$ the functions were defined in [4] as follows. Let $\nu$ be a positive integer, and let $n_{1}$ and $n_{2}$ be integers with $\left(n_{2}, j\right)=1$. We put

$$
\begin{gathered}
b_{m}\left(\nu, j, n_{1}, n_{2}\right)=(\pi / 8) \sum_{\substack{k=1 \\
k \equiv n_{1} j\left(\bmod j^{3}\right)}}^{\infty} k^{-1} A_{k, \nu}^{\left(n_{2}\right)}(m) . \\
(\nu / m)^{1 / 2} I_{1}\left(\frac{4 \pi \sqrt{m \nu}}{k}\right),
\end{gathered}
$$

where $I_{1}$ is the modified Bessel function of the first kind, defined by

and

$$
I_{1}(x)=\sum_{p=0}^{\infty} \frac{(x / 2)^{2 p+1}}{p !(p+1) !}
$$

$$
A_{k, \nu}^{\left(n_{2}\right)}(m)=\sum_{\substack{0 \leq h<k \\(h, k)=1 \\ h \equiv n_{2}(\bmod k)}} \exp \left[-\frac{2 \pi i}{k}\left(\nu h^{\prime}+m h\right)\right]
$$

is a generalized Kloosterman sum. Here $h^{\prime}$ is any integral solution of the congruence $h h^{\prime} \equiv-1(\bmod k)$. We now define

$$
a_{m}\left(\nu, j, n_{1}, n_{2}\right)=a_{m}=b_{m}\left(\nu, j, n_{1}, n_{2}\right)+b_{m}\left(\nu, j,-n_{1},-n_{2}\right) .
$$

For the moment we omit the cases $n_{1} \equiv 0, n_{2} \equiv 1(\bmod j)$ and $n_{1} \equiv 0$, $n_{2} \equiv-1(\bmod j)$, and put

$$
\lambda_{\nu}\left(j, n_{1}, n_{2} ; \tau\right)=\sum_{m=1}^{\infty} a_{m}\left(\nu, j, n_{1}, n_{2}\right) e^{2 \pi i m \tau / j} .
$$

Received November 28, 1960. The work on this paper was begun while the author was a National Science Foundation fellow at the California Institute of Technology during the summer of 1960. The work was completed under the support of National Science Foundation grant number 484-3305 at the University of Wisconsin. I would like to thank Dr. Morris Newman of the National Bureau of Standards for a very helpful conversation during which some of the ideas of this paper crystallized. 
For the cases $n_{1} \equiv 0, n_{2} \equiv 1(\bmod j)$ and $n_{1} \equiv 0, n_{2} \equiv-1(\bmod j)$ we make the separate definition

$$
\lambda_{\nu}\left(j, n_{1}, n_{2} ; \tau\right)=\frac{1}{16} e^{-2 \pi i \nu \tau / j}+\sum_{m=1}^{\infty} a_{m}\left(\nu, j, n_{1}, n_{2}\right) e^{2 \pi i m \tau / j},
$$

with $a_{m}\left(\nu, j, n_{1}, n_{2}\right)$ defined by (1.2). Notice that in the Definitions (1.3) and (1.4), $n_{1}$ and $n_{2}$ enter only modulo $j$; also, $\lambda_{\nu}\left(j, n_{1}, n_{2} ; \tau\right)=\lambda_{\nu}\left(j,-n_{1}\right.$, $\left.-n_{2} ; \tau\right)$, so that, with fixed $\nu$, we need only consider the $j \cdot(\phi(j) / 2)$ functions with $0 \leqq n_{1}<j, 0 \leqq n_{2}<j / 2$.

The following theorem is the fundamental one of [4] since it summarizes the transformation properties of $\lambda_{\nu}\left(j, n_{1}, n_{2} ; \tau\right)$ under $G(j)$.

THEOREM (1.5). The function $\lambda_{\nu}\left(j, n_{1}, n_{2} ; \tau\right)$ is regular in $\mathscr{J}(\tau)>0$ and there satisfies the transformation equations

$$
\lambda_{\nu}\left(j, n_{1}, n_{2} ; V \tau\right)=\lambda_{\nu}\left(j, n_{1}, n_{2} ; \tau\right)+\omega_{\nu}\left(j, n_{1}, n_{2} ; V\right),
$$

for all $V=\left(\begin{array}{l}a b \\ c d\end{array}\right) \in G(j)$. Here $\omega_{2}\left(j, n_{1}, n_{2} ; V\right)$ does not depend on $\tau, a$, or $b$.

We should point out that in [4] this theorem is proved in detail only for the case $n_{1}=n_{2}=1$. However, the proof for the other cases is identical except when $n_{1}=0, n_{2}=1$, in which case minor changes have to be made.

Let $\mathscr{F}$, denote any fundamental region of $G(j)$. From the Definitions (1.3) and (1.4) the behavior of the functions $\lambda_{\nu}\left(j, n_{1}, n_{2} ; \tau\right)$ at the parabolic cusp at $\infty$ is clear. However, $\infty$ is the only parabolic cusp of $\mathscr{F}_{3}$ for which this is so. One of the principal objects of this paper, in fact, is to determine the behavior of these functions at the cusps other than that at $\infty$. It turns out (see $\S 3$ ) that $\lambda_{\nu}\left(j, n_{1}, n_{2} ; \tau\right)$ has a pole of order $\nu$ (in the appropriate uniformizing variable) at the one parabolic cusp of $\mathscr{F}_{j}$ of the form $P=j m_{0} / k_{0}$, with $k_{0} \equiv n_{1} j\left(\bmod j^{2}\right)$, $m_{0} \equiv n_{2}(\bmod j)$, and $\left(k_{0}, m_{0}\right)=1$; at all other parabolic cusps of $\mathscr{F}_{\text {, }}$ $\lambda_{\nu}\left(j, n_{1}, n_{2} ; \tau\right)$ is regular in the uniformizing variable. This result and Theorem (1.5) together show that $\lambda_{v}\left(j, n_{1}, n_{2} ; \tau\right)$ is an abelian integral.

In order to derive this result we first determine the behavior of the functions $\lambda_{2}\left(j, n_{1}, n_{2} ; \tau\right)$ under the group of transformations $G^{0}(j)$. This group consists of those elements $\left(\begin{array}{l}a b \\ c d\end{array}\right)$ of $G(1)$, the full modular group, satisfying the additional requirement that $b \equiv 0(\bmod j)$. The result, which is contained in $\S 2$, is of some interest in itself and is thus stated as a separate theorem.

2. Behavior under $G^{0}(j)$. In order to determine the behavior of the functions (1.3), (1.4) under $G^{0}(j)$ we make essential use of the methods and results of [4]. In particular the computations of sections 
3 and 4 of [4] show that, for $\tau$ on the positive imaginary axis, $\lambda_{\nu}\left(j, n_{1}, n_{2} ; \tau\right)$ can be written in the two forms (2.1) and (2.2), below. These two forms, while different in structure, are obtained by the same general method.

Suppose $a<0, b<0$, and $d>j c>0$. Put $t=j\left(c-\left(\frac{1}{2} b\right)\right) d^{-1}$ and let $\mathscr{T}(K)$ denote the trapezoid in the $k-m$ plane bounded by the lines $k=0, m=(a k-K t) / j c, m=(b k / j \pm K) d^{-1}$. We introduce the notation

$$
\sum_{k}^{n_{1}} \sum_{m}^{n_{2}}=\sum_{k \equiv n_{1} j\left(\bmod j^{2)} j_{(m, k)=1}\right.} \sum_{\substack{m=1 \\(\bmod j)}}+\sum_{k \equiv-n_{1} j\left(\bmod j j^{2}\right)} \sum_{\substack{m \equiv=-n_{2}(\bmod j) \\(m, k)=1}}
$$

Then the computations of $\S \S 3$ and 4 of [4] show that, for $\tau$ on the positive imaginary axis, $\lambda_{\nu}\left(j, n_{1}, n_{2} ; \tau\right)$ is equal to

$$
\begin{aligned}
& \frac{1}{16} e^{-2 \pi i \nu \tau / j}+ A\left(n_{1}, n_{2}\right)+\frac{1}{16} \lim _{K \rightarrow \infty(k, m) \in Y^{\prime}(K)} \sum_{\substack{k>0 \\
n_{1}}}^{n_{2}} e^{-2 \pi i \nu m^{\prime} / k} \\
& \cdot\left(\exp \left[\frac{2 \pi i \nu}{k(k \tau / j-m)}\right]-1\right), \quad \text { if } n_{1}=0, n_{2}=1
\end{aligned}
$$

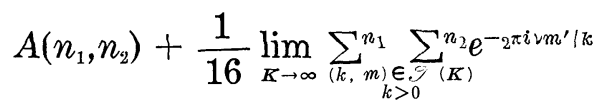

$$
\begin{aligned}
& \left(\exp \left[\frac{2 \pi i \nu}{k(k \tau / j-m)}\right]-1\right), \quad \text { otherwise. }
\end{aligned}
$$

These same methods can be used to show that, for $\tau$ on the positive imaginary axis, $\lambda_{\nu}\left(j, n_{1}, n_{2} ; \tau\right)$ is equal to

$$
\begin{aligned}
\frac{1}{16} e^{-2 \pi i \nu \tau / j}+ & A\left(n_{1}, n_{2}\right)+\frac{1}{16} \lim _{K \rightarrow \infty} \sum_{k=1}^{[t K]} \sum_{|m| \leqq K}^{n_{2}} e^{-2 \pi i \nu m^{\prime} / k} . \\
& \left(\exp \left[\frac{2 \pi i \nu}{k(k \tau / j-m)}\right]-1\right), \quad \text { if } n_{1}=0, n_{2}=1
\end{aligned}
$$

$$
\begin{aligned}
A\left(n_{1}, n_{2}\right)+\frac{1}{16} \lim _{K \rightarrow \infty} \sum_{k=1}^{[t K]} \sum_{1} \sum_{|m| \leqq K}^{n_{2}} e^{-2 \pi i \nu m^{\prime} / k} . & \\
& \left(\exp \left[\frac{2 \pi i \nu}{k(k \tau / j-m)}\right]-1\right), \quad \text { otherwise. }
\end{aligned}
$$

In (2.1) and (2.2) $A\left(n_{1}, n_{2}\right)$ is independent of $\tau$. We could give an explicit expression for $A\left(n_{1}, n_{2}\right)$ in terms of $n_{1}, n_{2}, \nu$, and $j$, but this of no significance here.

Now, put

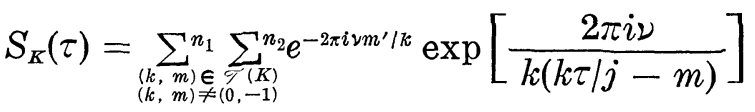

$$
\begin{aligned}
& =\sum_{\substack{(k, m) \in \mathcal{G} \\
(k, m) \neq(0,-1)}}^{n_{1}} \sum_{\substack{(K) \\
(k)}}^{n_{2}} \exp \left[2 \pi i \nu \frac{-k^{\prime}-m^{\prime} \tau / j}{k \tau / j-m}\right],
\end{aligned}
$$


where we have set $-k^{\prime}=\left(m m^{\prime}+1\right) / k$, or

$$
k k^{\prime}+m m^{\prime}+1=0 .
$$

Notice that in the finite sum defining $S_{K}(\tau)$, the summation condition $k>0$ does not appear, but that we have excluded the term for $(k, m)=(0,-1)$ from the sum. In the case $n_{1}=0, n_{2}=1$, the term corresponding to $k=0, m=1$ appears in the sum, and when $k=0, m=1$, we can choose $k^{\prime}=0, m^{\prime}=-1$. Here $\left(k^{\prime}, m^{\prime}\right)$ is interpreted to be any pair of integers satisfying (2.3). (It can easily be shown that the terms in $S_{K}(\tau)$ are unaffected by the ambiguity in the choice of the pair $\left(k^{\prime}, m^{\prime}\right)$ ). Thus when $n_{1}=0, n_{2}=1$, the term $e^{-2 \pi i v \tau / s}$ appears in the sum for $S_{K}(\tau)$, and we have absorbed the extra term $e^{-2 \pi i v \tau / s}$, which appears in (2.1) when $n_{1}=0, n_{2}=1$, into $S_{K}(\tau)$. In all other cases $k=0$ does not appear in the sum and the condition $(k, m) \neq(0,-1)$ is no extra restriction.

If the pair $(k, m)$ is replaced by $(-k,-m)$, the pair $\left(k^{\prime}, m^{\prime}\right)$ is replaced by $\left(-k^{\prime},-m^{\prime}\right)$ and the corresponding term in $S_{K}(\tau)$ is unchanged. Therefore if we extend the region of summation in $S_{K}(\tau)$ by reflecting $\mathscr{T}(K)$ through the origin, $S_{K}(\tau)$ is multiplied by 2 . The new region of summation is the parallelogram $\mathscr{P}(K)$, bounded by the four lines

$$
m=\frac{b k}{j d} \pm \frac{K}{d}, m=(a k \pm K t) / j c
$$

At the same time, the new points $(k, m)$ that we have now introduced into the summation satisfy the proper congruence conditions on $k$ and $m$. Hence we may write

$$
S_{K}(\tau)=\frac{1}{2} \sum_{(k, m) \in \mathscr{\mathscr { P }}(\boldsymbol{K})}^{n_{1}} \sum_{n_{2}}^{n_{2}} \exp \left[2 \pi i \nu \frac{-k^{\prime}-m^{\prime} \tau / j}{k \tau / j-m}\right] .
$$

This expression for $S_{K}(\tau)$ is fundamental in deriving the transformation properties of our functions under $G^{0}(j)$.

Let $V=\left(\begin{array}{l}a b \\ c d\end{array}\right) \in G^{0}(j)$. That is $a, b, c$, and $d$ are integers with $a d-b c=1$ and $b \equiv 0(\bmod j)$. We shall further assume that $a<0$, $b<0$, and $d>j c>0$, so that $\lambda_{\nu}\left(j, n_{1}, n_{2} ; \tau\right)$ can be written in the form (2.1) with this particular choice of $a, b, c, d$. It follows from (2.4) that

$$
S_{K}(V \tau)=\frac{1}{2} \sum_{\left(k_{m} \in \mathscr{P}^{\prime}(K)\right.}^{n_{1}} \sum_{n_{i}}^{n_{i}} \exp \left[2 \pi i \nu \frac{-\left(d k^{\prime}+b m^{\prime} \mid j\right)-(\tau / j)\left(j c k^{\prime}+a m^{\prime}\right)}{(\tau / j)(a k-j c m)-(m d-b k / j)}\right] .
$$

If we now perform the transformation

$$
l=a k-j c m, n=-b k / j+m d,
$$

then the parallelogram $\mathscr{P}(K)$ in the $k-m$ plane is mapped onto the 
rectangle in the $l-n$ plane defined by $|l| \leqq t K,|n| \leqq K$. Furthermore, since $a d-b c=1$ and $b \equiv 0(\bmod j)$, it follows that the set of all $(k, m) \in \mathscr{P}(K)$ satisfying $(k, m)=1$ and $k \equiv n_{1} j\left(\bmod j^{2}\right), m \equiv n_{2}(\bmod j)$ is mapped in a one-to-one fashion onto the set of all pairs of integers $(l, n)$ in the rectangle satisfying $(l, n)=1$ and $l \equiv\left(a n_{1}-c n_{2}\right) j\left(\bmod j^{2}\right)$, $n \equiv d n_{2}(\bmod j)$. Also, the set of $(k, m) \in \mathscr{P}(K)$ satisfying $(k, m)=1$ and $k \equiv-n_{1} j\left(\bmod j^{2}\right), m \equiv-n_{2}(\bmod j)$ is mapped onto the set of $(l, n)$ in the rectangle satisfying $l \equiv-\left(a n_{1}-c n_{2}\right) j\left(\bmod j^{2}\right), n \equiv-d n_{2}(\bmod j)$. A straightforward computation shows that

$$
\begin{gathered}
(a k-j c m)\left(d k^{\prime}+b m^{\prime} / j\right)+(m d-b k / j)\left(j c k^{\prime}+a m^{\prime}\right)+1 \\
=k k^{\prime}+m m^{\prime}+1=0
\end{gathered}
$$

so that we may put

$$
l^{\prime}=d k^{\prime}+b m^{\prime} \mid j, n^{\prime}=j c k^{\prime}+a m^{\prime} .
$$

Hence (2.5) becomes

$$
S_{K}(V \tau)=\frac{1}{2} \sum_{|l| \leqq t K}^{a n_{1}-c n_{2}} \sum_{|n| \leqq K}^{a n_{2}} \exp \left[2 \pi i \nu \frac{-l^{\prime}-n^{\prime} \tau / j}{l \tau \mid j-n}\right],
$$

and finally, $S_{K}(V \tau)$ is equal to

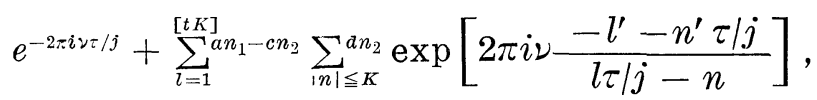

$$
\text { if } a n_{1}-c n_{2} \equiv 0, d n_{2} \equiv \pm 1(\bmod j)
$$

$$
\sum_{l=1}^{[t K]} a n_{1}-c n_{2} \sum_{|n| \leqq K}^{a n_{2}} \exp \left[2 \pi i \nu \frac{-l^{\prime}-n^{\prime} \tau / j}{l \tau / j-n}\right], \quad \text { otherwise . }
$$

In (2.6) we have separated out the terms correspoding to $l=0, n= \pm 1$ when those terms appear in the sum, that is, when $a n_{1}-c n_{2}$, $\equiv 0$, $d n_{2} \equiv \pm 1(\bmod j)$. Now it follows from $(2.1)$ and the definition of $S_{K}(\tau)$ that

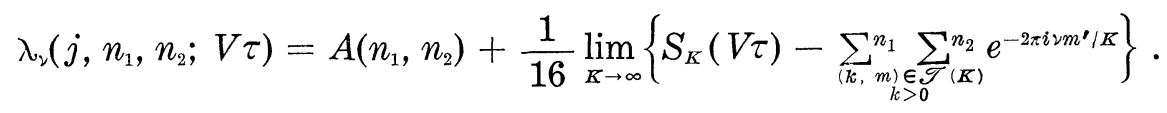

Thus, inserting (2.6) and comparing the result with (2.2), we find that

$$
\lambda_{\nu}\left(j, n_{1}, n_{2} ; V \tau\right)-\lambda_{\nu}\left(j, a n_{1}-c n_{2}, d n_{2} ; \tau\right)
$$

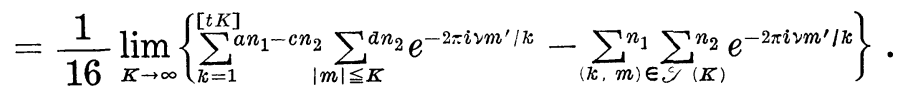


Now, let $V=\left(\begin{array}{l}a b \\ c d\end{array}\right)$ be any element of $G^{0}(j)$, that is, we remove the restrictions $a<0, b<0, d>j c>0$. Let $S=\left(\begin{array}{l}1 j \\ 01\end{array}\right) \in G^{0}(j)$. Cleary, if $p$ and $q$ are integers, then $S^{p}$ and $S^{q}$ are in $G^{0}(j)$, and a simple calculation yields

$$
S^{q} V S^{p}=\left(\begin{array}{ll}
\alpha & \beta \\
\gamma & \delta
\end{array}\right)
$$

with

$$
\begin{aligned}
\alpha & =a+q j c, & \beta & =p j(a+q j c)+b+q j d, \\
\gamma & =c, & \delta & =p j c+d .
\end{aligned}
$$

Now $p$ and $q$ can be chosen in such a way that $\alpha<0, \beta\langle 0, \delta>j \gamma>0$, so that we can apply (2.7) with $V$ replaced by $S^{q} V S^{p}$. Denoting the right hand side of $(2.7)$ by $\omega_{\nu}\left(j, n_{1}, n_{2} ; V\right)=\omega(V)$, we obtain

$$
\lambda_{\nu}\left(j, n_{1}, n_{2} ; S^{q} V S^{p} \tau\right)=\lambda_{\nu}\left(j, \alpha n_{1}-\gamma n_{2}, \delta n_{2} ; \tau\right)+\omega\left(S^{q} V S^{p}\right) .
$$

But

$$
\alpha \equiv \alpha, \beta \equiv b, \gamma \equiv c, \delta \equiv d(\bmod j)
$$

implies that

$$
\lambda_{\nu}\left(j, \alpha n_{1}-\gamma n_{2}, \delta n_{2} ; \tau\right)=\lambda_{\nu}\left(j, a n_{1}-c n_{2}, d n_{2} ; \tau\right) .
$$

On the other hand all the functions $\lambda_{\nu}$ defined by (1.3) are invariant under $S$. Thus,

$$
\lambda_{\nu}\left(j, n_{1}, n_{2} ; S^{q} V S^{p} \tau\right)=\lambda_{\nu}\left(j, n_{1}, n_{2} ; V S^{p} \tau\right),
$$

and we obtain

$$
\lambda_{\nu}\left(j, n_{1}, n_{2} ; V S^{p} \tau\right)=\lambda_{\nu}\left(j, a n_{1}-c n_{2}, d n_{2} ; \tau\right)+\omega\left(S^{q} V S^{p}\right) .
$$

In this equation we replace $\tau$ by $S^{-p} \tau$, again use the invariance of $\lambda_{\nu}$ under $S$, and thus obtain the following result.

THeOREM (2.8). If $V=\left(\begin{array}{l}a b \\ c d\end{array}\right) \in G^{0}(j)$, then for $\mathscr{J}(\tau)>0$

$$
\lambda_{\nu}\left(j, n_{1}, n_{2} ; V \tau\right)=\lambda_{\nu}\left(j, a n_{1}-c n_{2}, d n_{2} ; \tau\right)+\omega_{\nu}\left(j, n_{1}, n_{2} ; V\right),
$$

where $\omega_{\nu}\left(j, n_{1}, n_{2} ; V\right)$ is independent of $\tau$. Furthermore, if $S=\left(\begin{array}{l}1 j \\ 01\end{array}\right)$ and $p$ and $q$ are integers, then

$$
\omega_{\nu}\left(j, n_{1}, n_{2} ; S^{q} V S^{p}\right)=\omega_{\nu}\left(j, n_{1}, n_{2} ; V\right) .
$$

REMARK. The theorem has actually been proved only for purely imaginary $\tau$ since we derived (2.1) and (2.2) for such $\tau$ only. However the result follows immediately for $\mathscr{I}(\tau)>0$ by analytic continuation. 
It should be noted that (2.10) follows from the argument immediately preceding the statement of the theorem.

If we take $V \in G(j)$ in Theorem (2.8), then

$$
a n_{1}-c n_{2} \equiv n_{1}, d n_{2} \equiv n_{2}(\bmod j)
$$

and (2.9) becomes

$$
\lambda_{\nu}\left(j, n_{1}, n_{2} ; V \tau\right)=\lambda_{\nu}\left(j, n_{1}, n_{2} ; \tau\right)+\omega_{\nu}\left(j, n_{1}, n_{2} ; V\right) .
$$

Thus Theorem (1.5) is contained in Theorem (2.8).

It is easy to see that each $V \in G^{0}(j)$ simply permutes the set of $j \cdot \phi(j) / 2$ functions $\lambda_{\nu}$ (with $j$ and $\nu$ fixed). We will later show that, given any two of the $\lambda_{\nu}$ 's (with the same $j$ and $\nu$ ), there exists $V \in G^{0}(j)$ such that $V$ transforms one into the other in the sense of (2.9).

3. Behavior at the parabolic cusps. The parabolic cusps of $\mathscr{F}$, are the points in which $\mathscr{F}$, touches the real axis, and possibly the point at $\infty$. Every such point is either a rational point or $\infty$, and conversely, every rational point is a parabolic cusps for some $\mathscr{F}_{\text {j. }}$ By Theorem (1.5), the analytic behavior of $\lambda_{*}\left(j, n_{1}, n_{2} ; \tau\right)$ is the same at $V \tau$ as it is at $\tau$, for $V \in G(j)$. Thus in order to determine the behavior of our functions at the rational points we need only consider their behavior at the (finite number of) parabolic cusps of a single $\mathscr{F}_{g}$. However, it turns out to be just as convenient to examine directly the behavior of the functions at all the rational points and at $\infty$. We now regard $j, \nu, n_{1}$, and $n_{2}$ as fixed.

Let $P=r / s,(r, s)=1$, be any rational point. In this context we consider $\infty$ a rational point, since $\infty=1 / 0$. Now $P$ is the one fixed point of the parabolic transformation $V_{P} \in G(j)$, defined by

$$
V_{P}= \begin{cases}\left(\begin{array}{lr}
r s j+1 & -r^{2} j \\
s^{2} j & 1-r s j
\end{array}\right), \quad \text { if } P=r / s<\infty \\
\left(\begin{array}{ll}
1 & j \\
0 & 1
\end{array}\right), \quad \text { if } P=\infty,\end{cases}
$$

and we define $t_{P}$, the local uniformizing variable at $P$, by

$$
t_{P}=\left\{\begin{array}{l}
\exp \left[\frac{-2 \pi i}{s^{2} j(\tau-r / s)}\right], \quad \text { if } P=r / s<\infty \\
e^{2 \pi i \tau / j}, \quad \text { if } P=\infty .
\end{array}\right.
$$

It is an immediate consequence of Theorem (1.5) that

$$
\lambda_{\nu}\left(j, n_{1}, n_{2} ; V_{P} \tau\right)=\lambda_{\nu}\left(j, n_{1}, n_{2} ; \tau\right)+\omega_{\nu}\left(j, n_{1}, n_{2} ; V_{P}\right)
$$

and therefore $\lambda_{\nu}\left(j, n_{1}, n_{2} ; \tau\right)$ has an expansion at $P$ (or at $t_{P}=0$ ) of the 
form (cf., for example, [2, pp. 406-408])

$$
\lambda_{\nu}\left(j, n_{1}, n_{2} ; \tau\right)=\beta(P) \log t_{P}+\sum_{m=-\infty}^{\infty} \alpha_{m}(P) t_{P}^{m},
$$

where $\alpha_{m}(P)$ and $\beta(P)$ of course depend upon $j, \nu, n_{1}$, and $n_{2}$ in addition to $P$. Note that in the case $P=\infty$, the expansion (3.1) is already fully known, this being simply (1.3) or (1.4) by which $\lambda_{\nu}\left(j, n_{1}, n_{2} ; \tau\right)$ was originally defined. It is worth mentioning that $\beta(P)=0$ if and only if $\omega_{\nu}\left(j, n_{1}, n_{2} ; V_{P}\right)=0$.

Suppose $k_{0} \equiv n_{1} j\left(\bmod j^{2}\right), m_{0} \equiv n_{2}(\bmod j)$, and $\left(m_{0}, k_{0}\right)=1$. We shall determine the behavior of $\lambda_{\nu}\left(j, n_{1}, n_{2} ; \tau\right)$ at $P=m_{0} /\left(k_{0} / j\right)$, that is, the nature of the expansion (3.1) for such a $P$. Let $k_{0}^{\prime}$ and $m_{0}^{\prime}$ be chosen so that $k_{0} k_{0}^{\prime}+m_{0} m_{0}^{\prime}+1=0$, and put

$$
V_{k_{0}, m_{0}}=\left(\begin{array}{lr}
m_{0} & j k_{0}^{\prime} \\
k_{0} / j & -m_{0}^{\prime}
\end{array}\right) \in G^{0}(j) \text {. }
$$

Then, by Theorem (2.8) we have

$$
\begin{gathered}
\lambda_{\nu}\left(j, n_{1}, n_{2} ; V_{k_{0}, m_{\jmath}} \tau\right)=\lambda_{\nu}\left(j, m_{0} n_{1}-\frac{k_{0}}{j} n_{2},-m_{0}^{\prime} n_{2} ; \tau\right) \\
+\omega_{\nu}\left(j, n_{1}, n_{2} ; V_{k_{0}, m_{0}}\right) .
\end{gathered}
$$

Now $m_{0} n_{1}-k_{0} n_{2} / j \equiv 0(\bmod j)$ and $-m_{0}^{\prime} n_{2} \equiv-m_{0}^{\prime} m_{0}=k_{0} k_{0}^{\prime}+1 \equiv 1(\bmod j)$, and we have

$$
\lambda_{\nu}\left(j, n_{1}, n_{2} ; V_{k_{0}, m_{0}} \tau\right)=\lambda_{\nu}(j, 0,1 ; \tau)+\omega_{\nu}\left(j, n_{1}, n_{2} ; V_{k_{0}, m_{0}}\right) .
$$

Furthermore, $V_{k_{0}, m_{0}}^{-1}$ maps the point $P$ onto $\infty$. Therefore, since the expansion of $\lambda_{\nu}(j, 0,1 ; \tau)$ at $\infty$ is, by the definition (1.4)

$$
\lambda_{\nu}(j, 0,1 ; \tau)=(1 / 16) e^{-2 \pi i v \tau / j}+\sum_{m=1}^{\infty} a_{m} e^{2 \pi i m \tau / j},
$$

it follows from (3.2) that the expansion of $\lambda_{\nu}\left(j, n_{1}, n_{2} ; \tau\right)$ at $P=m_{0} /\left(k_{0} / j\right)$ has the form

$$
\lambda_{\nu}\left(j, n_{1}, n_{2} ; \tau\right)=\alpha_{-\nu} t_{P}^{-\nu}+\sum_{m=0}^{\infty} \alpha_{m}(P) t_{P}^{m} .
$$

We have derived part (i) of the following theorem.

TheOREM (3.4). (i) If $k_{0}$ and $m_{0}$ are integers such that $k_{0} \equiv n_{1} j$ $\left(\bmod j^{2}\right), m_{0} \equiv n_{2}(\bmod j)$, and $\left(m_{0}, k_{0}\right)=1$, then the expansion of $\lambda_{\nu}\left(j, n_{1}, n_{2} ; \tau\right)$ at $P=m_{0} /\left(k_{0} / j\right)$ is of the form (3.3).

(ii) If $P=r / s$ is any other rational point, then the expansion at $P$ has the form 


$$
\lambda_{\nu}\left(j, n_{1}, n_{2} ; \tau\right)=\sum_{m=0}^{\infty} \alpha_{m}(P) t_{P}^{m} .
$$

REMARKs. The proof of part (i) shows that for any two $\lambda_{\nu}$ 's with the same $\nu$ and $j$, there exists $V \in G^{0}(j)$ such that $V$ transforms one into the other. Given two rational points $P=r / s$ and $P^{\prime}=r^{\prime} / s^{\prime}$, with $(r, s)=1,\left(r^{\prime}, s^{\prime}\right)=1$, then $P$ and $P^{\prime}$ are equivalent under $G(j)$ (that is, there exists $V \in G(j)$ such that $V(P)=P^{\prime}$ ) if and only if $r \equiv r^{\prime}$, $s \equiv s^{\prime}(\bmod j)$. Thus in any $\mathscr{F}_{j}$ there is exactly one point of the type mentioned in part (i). The theorem shows that at this point $\lambda_{\nu}\left(j, n_{1}, n_{2} ; \tau\right)$ has a pole of order $\nu$ in the local uniformizing variable and that, at all other parabolic cusps, $\lambda_{2}\left(j, n_{1}, n_{2} ; \tau\right)$ is regular in the local variable. Thus, Theorems (1.5) and (3.4) together show that $\lambda_{\nu}\left(j, n_{1}, n_{2} ; \tau\right)$ is an abelian integral.

We now proceed with the proof of part (ii). Early in the computations of $\S 4$ of [4] an expression for $\lambda_{\nu}\left(j, n_{1}, n_{2} ; \tau\right)$ different from both (2.1) and (2.2) was obtained. In [4] this was used to derive (2.1) and (2.2). Here we find it convenient to make use of this earlier expression. In order to do this we introduce the notation

$$
\sum_{k}^{\#} \sum_{k \equiv n_{1} j\left(\bmod \rho^{2}\right)}, \quad \sum_{k}=\sum_{k \equiv-n_{1} j\left(\bmod j^{2}\right)}
$$

and

$$
\Sigma^{*}=\sum_{\substack{m \equiv n_{2}(\bmod j) \\(m, k)=1}}, \quad \sum_{*}=\sum_{\substack{m \equiv-n_{2}(\bmod j) \\(m, k)=1}} \cdot
$$

Then, for $\mathscr{F}(\tau)>0, \lambda_{\nu}\left(j, n_{1}, n_{2} ; \tau\right)$ is equal to

$$
\begin{array}{r}
\frac{1}{16} e^{-2 \pi \nu \tau / j}+A\left(n_{1}, n_{2}\right)+\frac{1}{16} \sum_{k=1}^{\infty} \lim _{N \rightarrow \infty|m| \leqq N} \sum^{*} e^{-2 \pi i \nu m^{\prime} \mid k}\left\{\exp \left[\frac{2 \pi i \nu}{k(k \tau / j-m)}\right]-1\right\} \\
+\frac{1}{16} \sum_{k=1}^{\infty} \lim _{N \rightarrow \infty|m| \leqq N} \sum_{*} e^{-2 \pi i \nu m^{\prime} \mid k}\left\{\exp \left[\frac{2 \pi i \nu}{k(k \tau / j-m)}\right]-1\right\}, \\
\text { if } n_{1} \equiv 0, n_{2} \equiv \pm 1(\bmod j)
\end{array}
$$

$$
\begin{gathered}
A\left(n_{1}, n_{2}\right)+\frac{1}{16} \sum_{k=1}^{\infty} \lim _{N \rightarrow \infty} \sum_{|m| \leqq N}^{*} e^{-2 \pi i \nu m^{\prime} \mid k}\left\{\exp \left[\frac{2 \pi i \nu}{k(k \tau / j-m)}\right]-1\right\} \\
+\frac{1}{16} \sum_{k=1} \lim _{N \rightarrow \infty} \sum_{|m| \leqq N} e^{-2 \pi i \nu m^{\prime} / k}\left\{\exp \left[\frac{2 \pi i \nu}{k(k \tau / j-m)}\right]-1\right\}, \quad \text { otherwise. }
\end{gathered}
$$

Now if $n_{1} \equiv 0, n_{2} \equiv \pm 1(\bmod j)$, it is clear that $\lambda_{\nu}\left(j, n_{1}, n_{2} ; \tau\right)$ has a pole of order $\nu$ at $\infty$ and this fact is included in part (i) of the theorem, if we choose $m_{0}=1, k_{0}=0$. In this case we need only consider the behavior of the function for finite rational points. At such points, however, $e^{-2 \pi i v \tau / J}$ is bounded, so that for all possible values of $n_{1}, n_{2}$ we 
may reduce the proof of part (ii) of the theorem to showing that the doubly infinite sums occurring in (3.6) behave decently at $P=r / s$.

We write

$$
\begin{aligned}
\sum_{k=1}^{\infty} \lim _{N \rightarrow \infty} \sum_{|m| \leqq N}^{*} e^{-2 \pi i \nu m^{\prime} \mid k}\left\{\exp \left[\frac{2 \pi i \nu}{k(k \tau / j-m)}\right]-1\right\} \\
=\sum_{k=1}^{\infty} \lim _{N \rightarrow \infty} \sum_{|m| \leqq N}^{*} e^{-2 \pi i \nu m^{\prime} \mid k} \frac{2 \pi i \nu}{k(k \tau / j-m)} \\
\quad+\sum_{k=1}^{\infty} \lim _{N \rightarrow \infty} \sum_{|m| \leqq N}^{*} e^{-2 \pi i \nu m^{\prime} / k} \sum_{p=2}^{\infty} \frac{1}{p !}\left(\frac{2 \pi i \nu}{k(k \tau \mid j-m)}\right)^{p} \\
=2 \pi i \nu \mathscr{S}_{1}(\tau)+\mathscr{S}_{2}(\tau) .
\end{aligned}
$$

The separation into two sums is justified in [4], where it is shown that the first sum is convergent. The second sum is absolutely convergent. as a triple sum. $\mathscr{S}_{2}(\tau)$ will be treated first.

Lemma (3.8). Let $P=r / s$ be as in part (ii) of Theorem (3.4), and let $\tau=r / s+i y(y>0)$. Then $\mathscr{S}_{2}(\tau)$ is bounded as $y \rightarrow 0+$. In fact,

$$
\left|\mathscr{S}_{2}(\tau)\right| \leqq \zeta(2)\left(2 e^{2 \pi \nu}+3 \varepsilon^{2 \pi \nu|s|}\right),
$$

on the half-line $\mathscr{R}(\tau)=r / s, \mathscr{J}(\tau)>0$.

Proof. From the definition of $\mathscr{S}_{2}(\tau)$ it follows that

$$
\left|\mathscr{S}_{2}(\tau)\right| \leqq \sum_{p=2}^{\infty} \frac{(2 \pi \nu)^{p}}{p !} \sum_{k=1}^{\infty} \frac{1}{k^{p}} \sum_{m=-\infty}^{\infty} \frac{1}{|k \tau / j-m|^{p}} .
$$

Now,

$$
|k \tau / j-m|=\sqrt{(k r / j s-m)^{2}+k^{2} y^{2} / j^{2}}>\left|\frac{k r}{j s}-m\right|
$$

and

$$
\left|\frac{k r}{j s}-m\right|=|s|^{-1}\left|\frac{k r}{j}-m s\right| \geqq|s|^{-1},
$$

since $k r / j-m s$ is an integer different from zero. For if $k r / j-m s=$ 0 , then $P=r / s=m /(k / j)$ and since $k \equiv n_{1} j\left(\bmod j^{2}\right), m \equiv n_{2}(\bmod j)$, $(m, k)=1$, this contradicts our choice of $P$. Therefore we may break up the innermost sum of (3.9) as follows:

$$
\sum_{m=-\infty}^{\infty} \frac{1}{|k \tau / j-m|^{p}}<\sum_{m<k r / s s}^{*}\left(\frac{k r}{j s}-m\right)^{-p}+\sum_{m>k r / s s}^{*}\left(m-\frac{k r}{j s}\right)^{-p} .
$$

But, 


$$
\begin{array}{r}
\sum_{m<k r / j s}^{*}\left(\frac{k r}{j s}-m\right)^{-p} \leqq \int_{-\infty}^{[k r / j s]-1}(k r / j s-x)^{-p} d x+2|s|^{p} \\
\leqq \frac{1}{p-1}+2|s|^{p} \leqq 1+2|s|^{p}, \quad \text { since } p \geqq 2 .
\end{array}
$$

Likewise,

$$
\begin{gathered}
\sum_{m>k r / s s}^{*}\left(m-\frac{k r}{j s}\right)^{-p} \int_{[k r / j s]+2}^{\infty}\left(x-\frac{k r}{j s}\right)^{-p} d x+|s|^{p} \\
\leqq \frac{1}{p-1}+|s|^{p} \leqq 1+|s|^{p}
\end{gathered}
$$

Therefore,

$$
\sum_{m=-\infty}^{\infty} \frac{1}{|k \tau| j-\left.m\right|^{p}}<2+3|s|^{p}
$$

Thus, by (3.9),

$$
\left|\mathscr{S}_{2}(\tau)\right|<2 \sum_{p=2}^{\infty} \frac{(2 \pi \nu)^{p}}{p !} \zeta(p)+3 \sum_{p=2}^{\infty} \frac{(2 \pi \nu|s|)^{p}}{p !} \zeta(p)<\zeta(2)\left(2 e^{2 \pi \nu}+3 e^{2 \pi \nu|s|}\right),
$$

since $\zeta(p) \leqq \zeta(2)$ follows from $p \geqq 2$, and the lemma is proved.

In order to treat $\mathscr{S}_{1}(\tau)$ we use a refinement of the method that was used in [4] to show that $\mathscr{S}_{1}(\tau)$ converges.

LEMMA (3.10). Let $\tau=x+i y$, where $y>0$ and $x$ is not a rational number of the form occurring in Theorem (3.4), part (i). (Note that $x$ is not necessarilly rational here.) Then,

$$
\mathscr{S}_{1}(\tau)=o\left(\frac{1}{1-e^{-2 \pi y / s}}\right), \quad \text { as } y \rightarrow 0+.
$$

Proof. Consider the inner sum in the definition of $\mathscr{S}_{1}(\tau)$. As in $\S 2$ of [4], we have

$$
\sum_{|m| \leqq N}^{*} \frac{e^{-2 \pi i \nu m^{\prime} / k}}{k(k \tau / j-m)}=k^{-1} \sum_{0 \leqq k<k}^{*} e^{-2 \pi i \nu h^{\prime} / k} \sum_{\substack{n \\|n k+h| \leqq N}} \frac{1}{k \tau / j-h-n k},
$$

where we have put $m=h+n k$. Hence

$$
\lim _{N \rightarrow \infty} \sum_{|m| \leqq N}^{*} \frac{e^{-2 \pi i \nu m^{\prime} / k}}{k(k \tau / j-m)}=k^{-2} \sum_{0 \leq n<k}^{*} e^{-2 \pi i h^{\prime} \nu / k} \lim _{N \rightarrow \infty} \sum_{\substack{n \\|n k+h| \leqq N}}(\tau / j-h / k-n)^{-1}
$$

$$
=\frac{\pi i}{k^{2}} \sum_{0 \leqq h<k}^{*} e^{-2 \pi i \nu h^{\prime} / k}-\frac{2 \pi i}{k^{2}} \sum_{0 \leqq n<k}^{*} e^{-2 \pi i h^{\prime} \nu / k}\left\{1-e^{2 \pi i(\tau / g-h / k)}\right\}^{-1} .
$$

In order to handle the sums on the right hand side of (3.11), we need 
the asymptotic estimate [5]

$$
\sum_{0 \leqq h<k}^{*} \exp \left[\frac{-2 \pi i}{k}\left(\nu h^{\prime}+p h\right)\right]=\mathscr{Q}\left(k^{2 / 3+\delta}\right), \quad \text { as } k \rightarrow \infty,
$$

where $\delta$ is any positive number, and the constant involved does not depend on $p$. Then,

(3.13) $\frac{\pi i}{k^{2}} \sum_{0 \leqq h<k}^{*} e^{-2 \pi i h^{\prime} \nu / k}=\frac{\pi i}{k^{2}} \sum_{0 \leqq h<k}^{*} \exp \left[\frac{-2 \pi i}{k}\left(\nu h^{\prime}+k h\right)\right]=\mathscr{Q}\left(k^{-4 / 3+\delta}\right)$,

as $k \rightarrow \infty$. We put

$$
\begin{gathered}
2 \pi i \sum_{k=1}^{\infty} \frac{1}{k^{2}} \sum_{0 \leqq h<k}^{*} e^{-2 \pi i h^{\prime} \nu / k}\left\{1-e^{2 \pi i(\tau / j-h / k)}\right\}^{-1} \\
=2 \pi i \sum_{k=1}^{M}+2 \pi i \sum_{k>M}^{\#},
\end{gathered}
$$

where $M$ is a positive integer to be specified. The second sum on the right hand side of (3.14) can be written

$$
2 \pi i \sum_{k>M}^{\#} \frac{1}{k^{2}} \sum_{0 \leq h<k}^{*} e^{-2 \pi i h^{\prime} \nu / k} \sum_{p=0}^{\infty} e^{2 \pi i p(\tau / g-h / k)} .
$$

But,

$$
\begin{gathered}
\frac{1}{k^{2}} \sum_{0 \leqq h<k}^{*} e^{-2 \pi i h^{\prime} \nu / k} \sum_{p=0}^{\infty} e^{2 \pi i p(\tau / j-h / k)} \\
=\frac{1}{k^{2}} \sum_{p=0}^{\infty} e^{2 \pi i p \tau / j} \sum_{0 \leqq h<k}^{*} \exp \left[\frac{-2 \pi i}{k}\left(\nu h^{\prime}+p h\right)\right],
\end{gathered}
$$

and therefore, by (3.12),

$$
\frac{1}{k^{2}} \sum_{0 \leqq h<k}^{*} e^{-2 \pi i h^{\prime} \nu / k}\left\{1-e^{2 \pi i(\tau / j-h / k)}\right\}^{-1}=Q\left(\frac{k^{-4 / 3+\delta}}{1-e^{-2 \pi y / j}}\right) .
$$

Hence, if we are given $\varepsilon>0$, there exists $M$ such that

$$
\left|\sum_{k>M l}^{\#} \frac{2 \pi i}{k^{2}} \sum_{0 \leqq h<k}^{*} e^{-2 \pi i h^{\prime} \nu / k}\left\{1-e^{2 \pi i(\tau / j-h / k)}\right\}^{-1}\right|<\frac{\varepsilon}{1-e^{-2 \pi y / j}} .
$$

Up to this point we have not used the special nature of $x$ in the proof. This will now be employed in estimating the first sum on the right hand side of (3.14). In this sum there are a finite number of different $h$ 's. Thus, only a finite number of pairs $(h, k)$ occur in the sum, and there exists a pair $\left(h_{1}, k_{1}\right)$ that minimizes the expression

$$
\left|1-e^{2 \pi i(\tau / /-h / k)}\right|
$$

for all pairs in this sum. Furthermore, since $y>0$, it is easy to see 
that

$$
\left|1-e^{2 \pi i\left(\tau / j-h_{1} / k_{1}\right)}\right|>0
$$

and we thus have

$$
\begin{gathered}
\left|\sum_{k=1}^{M} \# \frac{2 \pi i}{k^{2}} \sum_{0 \leqq h<k}^{*} e^{-2 \pi i h^{\prime} \nu / k}\left\{1-e^{2 \pi i(\tau / j-h / k)}\right\}^{-1}\right| \\
\leqq 2 \pi\left|1-e^{2 \pi i\left(\tau / \zeta-h_{1} / k_{1}\right)}\right|^{-1} \sum_{k=1}^{M} \frac{\phi(k)}{k^{2}} \\
<2 \pi\left|1-e^{2 \pi i\left(\tau / j-h_{1} / k_{1}\right)}\right|^{-1} \sum_{k=1}^{M} k^{-1},
\end{gathered}
$$

where $\phi(k)$ is the Euler function. But a simple geometric consideration shows that

$$
2\left|1-e^{2 \pi i\left(\tau / j-h_{1} / k_{1}\right)}\right| \geqq\left|1-e^{-2 \pi i\left(x / j-h_{1} / k_{1}\right)}\right|,
$$

since $\tau=x+i y$, with $y>0$. By the restriction on $x$ and the fact that $k_{1} \equiv n_{1} j\left(\bmod j^{2}\right), h_{1} \equiv n_{2}(\bmod j)$, and $\left(h_{1}, k_{1}\right)=1$, we have $x / j-h_{1} / k_{1} \neq 0$, and therefore

$$
\left|1-e^{2 \pi i\left(x / j-h_{1} / k_{1}\right)}\right|>0 .
$$

Using this in (3.16) and replacing the finite sum on $k$ by an integral, we obtain

$$
\begin{aligned}
\mid \sum_{k=1}^{M} & \frac{2 \pi i}{k^{2}} \sum_{0 \leq h<k}^{*} e^{-2 \pi i h^{\prime} \nu / k}\left\{1-e^{2 \pi i(\tau / j-h / k)}\right\}^{-1} \mid \\
& <4 \pi\left|1-e^{2 \pi i\left(x / j-h_{1} / k_{1}\right)}\right|^{-1} \log (M+1) .
\end{aligned}
$$

Combining (3.13), (3.15), and (3.17), we find that

$$
\begin{gathered}
\left(1-e^{-2 \pi y / j}\right)\left|\mathscr{S}_{1}(x+i y)\right| \leqq C\left(1-e^{-2 \pi y / j}\right)+\varepsilon \\
+\frac{4 \pi \log (M+1)}{\left|1-e^{2 \pi i\left(x / j-h_{1} / k_{1}\right)}\right|}\left(1-e^{-2 \pi y / j}\right),
\end{gathered}
$$

where $C$ is a positive constant. Now we have already chosen $M$ (depending on $\varepsilon$ ), and, given $\varepsilon$ and $M$, there exists $y_{0}>0$ such that, for $0<y<y_{0}$,

$$
C\left(1-e^{-2 \pi y / j}\right)+\frac{4 \pi \log (M+1)}{\left|1-e^{2 \pi i\left(x / j-h_{1} / k_{1}\right)}\right|}\left(1-e^{-2 \pi y / j)}<2 \varepsilon .\right.
$$

Therefore,

$$
\left(1-e^{-2 \pi y / j)} \mathscr{S}_{1}(x+i y) \rightarrow 0, \quad \text { as } y \rightarrow 0+,\right.
$$


and the proof of the lemma is complete.

We now return to the proof of Theorem (3.4), part (ii). If we go back to (3.7) we see that, with $\tau$ as specified in part (ii) of Theorem (3.4), lemmas (3.8) and (3.10) imply

$$
\begin{aligned}
\sum_{k=1}^{\infty} \lim _{N \rightarrow \infty} \sum_{|m| \leqq N}^{*} e^{-2 \pi i \nu m^{\prime} / k}\left\{\exp \left[\frac{2 \pi i}{k(k \tau / j-m)}\right]-1\right\} \\
=o\left(\frac{1}{1-e^{-2 \pi y / j}}\right), \quad \text { as } y \rightarrow 0+.
\end{aligned}
$$

The same method of proof yields

$$
\begin{aligned}
\sum_{k=1}^{\infty} \lim _{N \rightarrow \infty} \sum_{|m| \leq N} e^{-2 \pi i \nu m^{\prime} / k}\left\{\exp \left[\frac{2 \pi i \nu}{k(k \tau / j-m)}\right]-1\right\} \\
=o\left(\frac{1}{1-e^{-2 \pi y / j}}\right), \quad \text { as } y \rightarrow 0+.
\end{aligned}
$$

Looking at (3.6) we conclude that

$$
\lambda_{\nu}\left(j, n_{1}, n_{2} ; r / s+i y\right)=o\left(\frac{1}{1-e^{-2 \pi y / s}}\right), \quad \text { as } y \rightarrow 0+,
$$

or, what is the same,

$$
\lambda_{\nu}\left(j, n_{1}, n_{2} ; r / s+i y\right)=o\left(\frac{1}{y}\right), \quad \text { as } y \rightarrow 0+.
$$

Therefore, in (3.1), $\beta(P)=0$ and $\alpha_{m}(P)=0$, for $m<0$, and the proof of Theorem (3.4) is complete.

4. Construction of modular functions. It follows from Theorem (3.4) that for any parabolic $V \in G(j)$,

$$
\lambda_{\nu}\left(j, n_{1}, n_{2} ; V \tau\right)=\lambda_{\nu}\left(j, n_{1}, n_{2} ; \tau\right) ;
$$

that is for $V$ parabolic, the constant $\omega_{\nu}\left(j, n_{1}, n_{2} ; V\right)$ which occurs in Theorem (1.5) is zero. We can see this by noting that Theorem (3.4) implies that in the expansion about the fixed point of $V$, no logarithmic term appears. Therefore, if $G(j)$ is generated by parabolic $V$, the functions $\lambda_{\nu}\left(j, n_{1}, n_{2} ; \tau\right)$ are actually modular functions for $G(j)$; that is, they satisfy (4.1) for all $V \in G(j)$. If we now take $\nu=1$, recall the assumption $j \geqq 3$ of $\S 1$, choose $j \leqq 3$ so that $G(j)$ is generated by parabolic $V$, and let $n_{1}, n_{2}$ any permissible pair of integers, we have a function $\lambda_{1}\left(j, n_{1}, n_{2} ; \tau\right)$ which is a modular function for $G(j)$, and which, by Theorem (3.4) has exactly one pole of order one in $\mathscr{F}$, (cf. under Remarks). Thus, by the Riemann-Roch Theorem, $\mathscr{F}$, has genus zero 
and since $\mathscr{F}_{2}$ has genus zero, we may state the following result.

THEOREM (4.2). If $G(j)$, the principal congruence subgroup of level $j \geqq 2$, can be generated by parabolic transformations, then $\mathscr{F}$, has genus zero.

This theorem may very well be known. The converse has been known for some time. For $\mathscr{F}$, has a genus zero exactly when $j=2$, 3,4 , and $5[3$, p. 398], and in these cases $G(j)$ can indeed be generated by parabolic transformations [3, pp. 267, 354-356].

As was pointed out in $\S 5$ of [4], we can make use of Theorem (1.5) and the fact that $G(j)$ is finitely generated to construct modular functions for $G(j)$. This is accomplished by taking a linear combination of the $\lambda_{\nu}\left(j, n_{1}, n_{2} ; \tau\right)$, with $j$ fixed and (possibly) different $\nu$ and $n_{1}, n_{2}$, in such a way that the resulting linear combinations of the constants $\omega_{2}\left(j, n_{1}, n_{2} ; V\right)$ vanish for a set of $V$ which generate $G(j)$. In order to be assured that we can carry this through we take a linear combination of at least $q+1$ functions, where $q$ is the minimal number of generators for $G(j)$. (It is known that $q=2 q+\mu / j-1$, where $p$ is the genus of $\mathscr{F}_{j}$, and $\mu$ is the index of $G(j)$ in $G(1)$.) If we choose the functions in the linear combination so that they all have the same $n_{1}$ and $n_{2}$, the resulting linear combination has a pole at only one point of $\mathscr{F}_{j}$. On the other hand, we may also place poles at several different points of $\mathscr{F}$, by choosing functions with different $n_{1}, n_{2}$.

It has already been noted that when $3 \leqq j \leqq 5$, the $\lambda_{y}\left(j, n_{1}, n_{2} ; \tau\right)$ are themselves modular functions (no linear combination is necessary) because in these cases $G(j)$ can be generated by parabolic transformations. If we keep the condition $3 \leqq j \leqq 5$, and take $\nu=1$ and any permissible pair of integers $n_{1}, n_{2}$, we obtain a modular function $\lambda_{1}\left(j, n_{1}, n_{2} ; \tau\right)=\lambda_{1}$ for $G(j)$ with exactly one pole of order one in $\mathscr{F}_{1}$. By a well known theorem in the theory of modular functions [1, p. 97], it then follows that every modular function for $G(j)$ is a rational function of $\lambda_{1}$. In particluar, if $M \in G(1)$, then $\lambda_{1}\left(j, n_{1}, n_{2} ; M \tau\right)$ is again a modular function for $C(j)$ (since $G(j)$ is normal in $G(1)$ ), and thus is a rational function of $\lambda_{1}$. Now, the only pole of $\lambda_{1}$ in $\mathscr{F}_{,}$is a pole of order one at a parabolic cusp specified by Theorem (3.4). If we call this point $P$, the only pole of $\lambda_{1}\left(j, n_{1}, n_{2} ; M \tau\right)$ is a pole of order one at the parabolic cusp $M^{-1}(P)$. If $M^{-1}(P)$ is equivalent to $P$ under $G(j)$, then there exist complex numbers $A_{\mu}$ and $B_{\mu}$ such that

$$
\lambda_{1}\left(j, n_{1}, n_{2} ; M \tau\right)=A_{\mu} \lambda_{1}+B_{M} .
$$

If $M^{-1}(P)$ is not equivalent to $P$ under $G(j)$, then there are complex numbers $A_{M}$ and $B_{M}$ such that 


$$
\lambda_{1}\left(j, n_{1}, n_{2} ; M \tau\right)=\frac{A_{M}}{\lambda_{1}-\lambda_{1}\left(j, n_{1}, n_{2} ; M^{-1}(P)\right)}+B_{M} .
$$

We summarize in part (i) of the following theorem.

THeORem 4.3. (i) Let $3 \leqq j \leqq 5$. If $M \in G(1)$, then there exist complex numbers $\alpha_{M}, \beta_{M}, \gamma_{M}, \delta_{M}$ such that

$$
\lambda_{1}\left(j, n_{1}, n_{2} ; M \tau\right)=\frac{\alpha_{M} \lambda_{1}+\beta_{M}}{\gamma_{M} \lambda_{1}+\delta_{M}} \equiv M^{*}\left(\lambda_{1}\right) .
$$

(ii) The set of all $M^{*}$ regarded as linear fractional transformations, obtained by letting $M$ run through $G(1)$, is a finite group; the mapping $M \rightarrow M^{*}$ defined by (4.4) is a homomorphism of $G(1)$ onto this group, with kernel $G(j)$. Thus the group in question is isomorphic to $G(1) / G(j)$.

Proof of (ii). The mapping $M \rightarrow M^{*}$ is obviously a homomorphism and thus the set of $M^{*}$ forms a group. If we can show that the kernel is $G(j)$, the remainder of the theorem follows. Since $\lambda_{1}$ is a modular functions for $G(j)$, the kernel obviously contains $G(j)$. To show that the kernel is contained in $G(j)$, we choose $M \notin G(j)$ and show that $M$ cannot be in the kernel.

We first remark that since $\lambda_{1}$ is a modular function with one pole in $\mathscr{F}_{j}$, it follows from [1, p. 94, Theorem 11] that $\lambda_{1}$ assumes every complex value exactly once in $\mathscr{F}_{j}$. Let $M \notin G(j)$. If $M$ is in the kernel then $\lambda_{1}\left(j, n_{1}, n_{2} ; M \tau\right)=\lambda_{1}$. But since $M \notin G(j)$ there are distinct points $\tau_{1}$ and $\tau_{2}$, not equivalent under $G(j)$, such that $\tau_{2}=M \tau_{1}$. It follows that there are two distinct points of $\mathscr{F}_{j}$, where $\lambda_{1}$ assumes the same value. This is a contradiction, so that $M$ is not in the kernel, and the proof is complete.

REMARK. The group of $M^{*}$ 's apparently depends upon the choice of $n_{1}$ and $n_{2}$. The theorem of course shows that for all choices of $n_{1}$ and $n_{2}$ (with $j$ fixed) the groups obtained are isomorphic. It is not yet clear whether these groups are actually identical, in the sense that they contain precisely the same linear fractional transformations $M^{*}$. In order to decide this it appears necessary to determine the values of the functions $\lambda_{y}\left(j, n_{1}, n_{2} ; \tau\right)$ at all of the parabolic cusps of $\mathscr{F}_{j}$.

5. The case $j=2$. Up until now the case $j=2$ has been omitted from the discussion, because its inclusion would have made certain computations more cumbersome and complicated unnecessarily the statements of theorems. We now treat this case for the sake of completeness and because it affords a simple example of the situation described above. 
Since $j=2$, there are only two possible choices for the pair $\left(n_{1}, n_{2}\right)$, namely $(0,1)$ and $(1,1)$. Accordingly, we define

$$
\begin{gathered}
\lambda_{\nu}(2,1,1 ; \tau)=\sum_{m=1}^{\infty} b_{m}(\nu, 2,1,1) e^{\pi i m \tau} \\
\lambda_{\nu}(2,0,1 ; \tau)=\frac{1}{16} e^{-2 \pi i \nu \tau}+\sum_{m=1}^{\infty} b_{m}(\nu, 2,0,1) e^{\pi i m \tau},
\end{gathered}
$$

where $b_{m}(\nu, 2,1,1)$ and $b_{m}(\nu, 2,0,1)$ are defined by (1.1). The techniques of the previous sections yield the following results.

(i) The functions $\lambda_{\nu}\left(2, n_{1}, n_{2} ; \tau\right)$ are regular in $\mathscr{J}(\tau)>0$ and there satisfy

$$
\lambda_{\nu}\left(2, n_{1}, n_{2} ; V \tau\right)=\lambda_{\nu}\left(2, n_{1}, n_{2} ; \tau\right), \quad \text { for } V \in G(2) .
$$

No additive constant appears on the right hand side since, as previously remarked, $G(2)$ is generated by parabolic transformations. then

(ii) If $V=\left(\begin{array}{l}a b \\ c d\end{array}\right)$ with $a d-b c=1$ and $a \equiv c \equiv d \equiv 1, b \equiv 0(\bmod 2)$,

$$
\begin{aligned}
& \lambda_{\nu}(2,1,1 ; V \tau)=\lambda_{\nu}(2,0,1 ; \tau)+\omega_{\nu}(2,1,1 ; V) \\
& \lambda_{\nu}(2,0,1 ; V \tau)=\lambda_{\nu}(2,1,1 ; \tau)+\omega_{\nu}(2,0,1 ; V) .
\end{aligned}
$$

(iii) We can choose an $\mathscr{F}_{2}$ which has as parabolic cusps the points $-1,0$, and $\infty$ [1, p. 82]. The function $\lambda_{\nu}(2,1,1 ; \tau)$ is zero at $\infty$, regular at 0 , and has a pole of order $\nu$ at -1 . The function $\lambda_{\nu}(2,0,1 ; \tau)$ is regular at -1 and 0 , and has a pole of order $\nu$ at $\infty$. Note that $\lambda_{1}(2,1,1 ; \tau)=\lambda(\tau)$ and $\lambda_{1}(2,0,1 ; \tau)=\mu(\tau)=1 / \lambda(\tau)$, where $\lambda(\tau)$ and $\mu(\tau)$ are the classical modular functions connected with $G(2)$. The expressions (5.1) for these functions were first given by Simons [6].

(iv) Theorem (4.3) holds when $j=2$. In this case the matrices $M^{*}$ are completely known [6], and the groups occurring in Theorem (4.3), part (ii) for $\left(n_{1}, n_{2}\right)=(1,1)$ and $\left(n_{1}, n_{2}\right)=(0,1)$ are actually identical. It does not appear to be easy to prove this by the methods of this paper.

6. Conclusion. In [4] we constructed modular forms, of positive even integral dimension and multiplier system identically one, for $G(j)$. In this paper nothing has been said about the behavior of these forms at the parabolic cusps of $\mathscr{F}_{3}$, since in a future publication, forms of arbitrary nonnegative integral dimension with more arbitrary multiplier systems for $G(j)$ will be constructed and the behavior of these forms at the cusps will be discussed there.

It is of interest to note that the methods and results of this paper and [4] can be applied to construct modular functions and forms for 
certain other congruence subgroups of the modular group.

\section{REFERENCES}

1. L. R. Ford, Automorphic functions, New York, Chelsea, 1951.

2. E. Hecke, Mathematische Werke, Göttingen, Vandenhoeck \& Ruprecht, 1959.

3. F. Klein and R. Fricke, Vorlesungen über die Theorie der Elliptischen Modulfunktionen, vol. 1, Leipzig, 1890-92.

4. M. I. Knopp, Construction of a class of modular functions and forms, Pacific J. Math., 11 (1961), 257-293.

5. H. Salié, Zur abschätzung der Fourierkoeffizienten ganzer Modulformen, Math. Zeit., 36 (1933), 263-278

6. W. H. Simons, The Fourier coefficients of the modular function $\lambda(\tau)$, Canadian J. Math., 4 (1952), 67-80.

The California Institute of Technology and The University Wisconsin 


\title{
PACIFIC JOURNAL OF MATHEMATICS
}

\author{
EDITORS
}

\author{
Ralph S. Phillips \\ Stanford University \\ Stanford, California \\ F. H. Brownell \\ University of Washington \\ Seattle 5, Washington
}

\author{
A. L. Whiteman \\ University of Southern California \\ Los Angeles 7, California \\ L. J. PAige \\ University of California \\ Los Angeles 24, California
}

ASSOCIATE EDITORS
E. F. BECKENBACH
D. DERRY
H. L. ROYDEN
E. G. STRAUS
T. M. CHERRY
M. OHTSUKA
E. SPANIER
F. WOLF

\section{SUPPORTING INSTITUTIONS}

\author{
UNIVERSITY OF BRITISH COLUMBIA \\ CALIFORNIA INSTITUTE OF TECHNOLOGY \\ UNIVERSITY OF CALIFORNIA \\ MONTANA STATE UNIVERSITY \\ UNIVERSITY OF NEVADA \\ NEW MEXICO STATE UNIVERSITY \\ OREGON STATE COLLEGE \\ UNIVERSITY OF OREGON \\ OSAKA UNIVERSITY \\ UNIVERSITY OF SOUTHERN CALIFORNIA
}

\author{
STANFORD UNIVERSITY \\ UNIVERSITY OF TOKYO \\ UNIVERSITY OF UTAH \\ WASHINGTON STATE COLLEGE \\ UNIVERSITY OF WASHINGTON \\ * * * * \\ AMERICAN MATHEMATICAL SOCIETY \\ CALIFORNIA RESEARCH CORPORATION \\ HUGHES AIRCRAFT COMPANY \\ SPACE TECHNOLOGY LABORATORIES \\ NAVAL ORDNANCE TEST STATION
}

Mathematical papers intended for publication in the Pacific Journal of Mathematics should be typewritten (double spaced), and the author should keep a complete copy. Manuscripts may be sent to any one of the four editors. All other communications to the editors should be addressed to the managing editor, L. J. Paige at the University of California, Los Angeles 24, California.

50 reprints per author of each article are furnished free of charge; additional copies may be obtained at cost in multiples of 50 .

The Pacific Journal of Mathematics is published quarterly, in March, June, September, and December. The price per volume (4 numbers) is $\$ 12.00$; single issues, $\$ 3.50$. Back numbers are available. Special price to individual faculty members of supporting institutions and to individual members of the American Mathematical Society: $\$ 4.00$ per volume; single issues, $\$ 1.25$.

Subscriptions, orders for back numbers, and changes of address should be sent to Pacific Journal of Mathematics, 103 Highland Boulevard, Berkeley 8, California.

Printed at Kokusai Bunken Insatsusha (International Academic Printing Co., Ltd.), No. 6, 2-chome, Fujimi-cho, Chiyoda-ku, Tokyo, Japan.

\section{PUBLISHED BY PACIFIC JOURNAL OF MATHEMATICS, A NON-PROFIT CORPORATION}

The Supporting Institutions listed above contribute to the cost of publication of this Journal, but they are not owners or publishers and have no responsibility for its content or policies. 


\section{Pacific Journal of Mathematics}

\section{Vol. 11, No. 2 December, 1961}

Tsuyoshi Andô, Convergent sequences of finitely additive measures........

Richard Arens, The analytic-functional calculus in commutative topological algebras..........................................

Michel L. Balinski, On the graph structure of convex polyhedra in

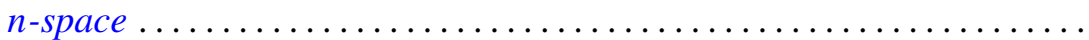

R. H. Bing, Tame Cantor sets in $E^{3}$...

Cecil Edmund Burgess, Collections and sequences of continua in the plane.

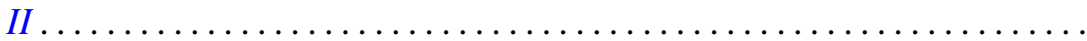

J. H. Case, Another 1-dimensional homogeneous continuum which contains an $\operatorname{arc}$

Lester Eli Dubins, On plane curves with curvature ................. 471

A. M. Duguid, Feasible flows and possible connections .............. 483

Lincoln Kearney Durst, Exceptional real Lucas sequences ................ 489

Gertrude I. Heller, On certain non-linear opeartors and partial differential equations........................................

Calvin Virgil Holmes, Automorphisms of monomial groups

Wu-Chung Hsiang and Wu-Yi Hsiang, Those abelian groups characterized by their completely decomposable subgroups of finite rank ..........

Bert Hubbard, Bounds for eigenvalues of the free and fixed membrane by finite difference methods .........................

D. H. Hyers, Transformations with bounded mth differences. .

Richard Eugene Isaac, Some generalizations of Doeblin's decomposition

John Rolfe Isbell, Uniform neighborhood retracts ..........

Jack Carl Kiefer, On large deviations of the empiric D. F. of vector chance variables and a law of the iterated logarithm...

Marvin Isadore Knopp, Construction of a class of modular functions and forms. II. . .

Gunter Lumer and R. S. Phillips, Dissipative operators in a Banach space....

Nathaniel F. G. Martin, Lebesgue density as a set function ...

Shu-Teh Chen Moy, Generalizations of Shannon-McMillan theorem ...

Lucien W. Neustadt, The moment problem and weak convergence in $L^{2}$

Kenneth Allen Ross, The structure of certain measure algebras...

James F. Smith and P. P. Saworotnow, On some classes of scalar-product algebras.

Dale E. Varberg, On equivalence of Gaussian measures. . 